\title{
Case Reports: Yes or No
}

\author{
Dolores Buscemi MD, Cihan Cevik MD
}

In April the American Journal of Medical Sciences made a decision to no longer publish Case Reports and to publish abbreviated Case Letters. Many other prominent journals have also stopped publishing case reports. Case reports have been published for at least two centuries, ${ }^{1}$ but this form of medical literature and its contribution have come under increased critical examination. Some editors suggest that they do not contribute much to an already extensive medical literature and that they potentially diminish the reputation of the journal. Is there sufficient value in publishing case reports to make it worth the time investment for authors and journals given the uncertain return?

\section{Yes}

We think that they add value to medical literature for the following reasons. Case reports can provide important information about a new disease. For example, Vandenbroucke notes that a case series and astute observations led to the identification of the West Nile Virus. ${ }^{2}$ Case reports can also provide information about new associations among diseases, about new treatment approaches, and about new complications associated with either a disease or treatment. The discovery of penicillin was first written about as a case report. ${ }^{3}$ Case reports with the associated literature review can remind clinicians about unusual medical disorders. We have had several patients in our hospital for whom reviewing the literature identified case reports directly related to the patients we had questions about and contributed to their care. Consequently, case reports are potentially unique sources of information which may be quite significant for some patients.

Case reports advance the clinical skills of authors. This is especially important for physicians in training. Case reports require a systematic case analysis, a careful literature review, and an organized and focused written presentation of the relevant facts in the case and the medical literature. This helps authors understand the details and deficiencies underlying all medical literature. Case reports provide the basis (data) for clinical reviews on uncommon clinical topics. They stimulate the development of both retrospective and prospective studies of clinical material available in most health care organizations. Finally, they can lead to clinical trials. A recent study in The Lancet concluded that eleven $(17 \%)$ of the case reports published in The Lancet led to clinical trials. ${ }^{4}$

\section{No}

Although case reports often describe novel, interesting, and sometimes heroic treatment for uncommon clinical situations, these therapies are usually uncontrolled and not evidence based. The described strategy may be ineffective and possibly harmful in a similar situation in a different setting. ${ }^{5}$ In addition, the described strategy is usually dependent on the personal skill and expertise of the reporting physician or team. The report may lead other physicians to try the described strategy in a presumed "similar" scenario and cause the physicians to overlook conventional but proven management. Furthermore, the immediate results from a case report often rely on subjective findings, and the likelihood of Type 1 error is high. The safety as well as the long term outcomes of these "salvage" strategies are usually unknown. The validity of the described report also depends heavily on the expertise of the reviewers of the manuscript. Safety or efficacy of the therapy can be easily overlooked by the reviewers since these clinical scenarios are too uncommon for the reviewers to have meaningful expertise. Finally, since the reports are often striking and unusual, the reviewers and journal editors may be naturally biased toward publication of the paper. 
Case reports unfortunately dilute the medical literature and may cause more important research studies, clinical trials, or guidelines to go unnoticed. ${ }^{6}$ By being short and easy to read case reports usually grab the attention of the reader like the sports section of the newspapers and leave longer and more detailed research studies ignored. Writing a case report is much easier than writing a research article. It usually does not require comprehensive preparation, approval, or consent unlike a research study. Many authors choose to work on a case report to get a "publication" rather than work on a more cumbersome research project which may need substantial effort even before the initiation of the study. Case report publications provide some "unfair" advantages to the clinicians over basic scientists who have limited opportunity to come across an unusual clinical scenario and publish it. This advantage/disadvantage will reflect on the "total number of scientific publications" of an academician and may lead to further inequality between clinicians and basic scientists. Similarly, some clinical specialties, such as surgery, cardiology, and radiology, have more opportunity to try a new surgical technique, observe an uncommon complication and potentially life-saving treatment, or encounter very uncommon pathology or anatomical variant, respectively, than other subspecialties.

Publication ethics and guidelines are less strict and difficult to apply to case reports and clinical image publications. An interesting clinical condition can be reported repeatedly if a similar uncommon condition is seen elsewhere by other physicians. ${ }^{2}$ Interestingly, the same patient can be reported more than one time if the patient is seen by multiple physicians, or if the patient sees another doctor after a period of time. This can be avoided if authors, reviewers, and journal editors have enough expertise and do comprehensive literature searches. However, this is usually not the case, and some journals have no alternative but publish case reports since they do not receive many research study submissions.

\section{Solutions}

Case reports obviously have some negative features. They increase work load for editors and reviewers. They potentially do not contribute much to an extensive and already available medical literature. The volume of medical literature is overwhelming, and readers cannot read all published case reports. These potential problems may be unavoidable but can be minimized. The authors, reviewers, and editors should be careful while reporting a particular case. Comprehensive research and review of the existing information should be performed by all parties. Pre-clinical data should be considered, when relevant. The limitations of the report must be described clearly in the abstract and manuscript. Clinical societies and practicing physicians should be encouraged to review these reports and share their opinions about the conclusions and implications of the reports. Journals might establish easy to use case report forums and solicit feedback. In addition, editors might refer articles with drug complications to the US Food and Drug Administration's MedWatch (the FDA Safety Information and Adverse Event reporting Program); this would potentially increase their impact on patient care.

Corresponding Author: Dolores Buscemi MD

Author Contact Information: Dolores.buscemi@ttuhsc.edu DOI: 10.12746/swrccc2013.0103.025

\section{REFERENCES}

1. Garrison FH.An Introduction to the History of Medicine.4th edition.W. B. Saunders, Philadelphia; 192.

2. Vandenbroucke JP. In defense of case reports and case series. Ann Intern Med 2001; 134:330-4. 
3. Neely JG, Karni RJ, Nussenbaum B, Paniello RC, Fraley PL, Wang EW, et al. Practical guide to understanding the value of case reports. Otolaryngol Head Neck Surg. 2008; 138:261-4.

4. Albrecht J, Meves A, Bigby M. Case reports and case series from Lancet had significant impact on medical literature. $J$ Clin Epidemiol 2005; 58:1227-32.

5. Angelini P, Uribe C, Monge J, Escobar JM, Hernandez-Vila E. Apical hypertrophic cardiomyopathy: preliminary attempt at palliation with use of subselective alcohol ablation. Tex Heart Inst $J$ 2012; 39:750-5.

6. Kounis Syndrome [online], http://www.ncbi.nlm.nih.gov/ pubmed/?term=kounis+syndrome, accessed June 2, 2013.

7. Cevik C, Izgi C, Boztosun B. A rare consequence of uncorrected atrial septal defect: diffuse pulmonary artery aneurysms. Tex Heart Inst $J$ 2004; 31:328-9.

8. Tartan Z, Cam N, Ozer N, Kaşikçioğlu H, Uyarel H. Giant pulmonary artery aneurysm due to undiagnosed atrial septal defect associated with pulmonary hypertension.Anadolu Kardiyol

Derg 2007; 7:202-4. 